Running head: STB

\title{
Re-Examining the Latent Structure of Suicidal Thoughts Using Taxometric Analysis: Implications for Testing Ideation to Action Theoretical Models of Suicidal Thoughts and Behavior
}

Andy P. Siddaway, Jill Holm-Denoma, Tracy K. Witte, John Ruscio

\author{
Author affiliations \\ Andy P. Siddaway, DClinPsy, Ph.D, Clinical Psychologist \\ Institute of Health \& Wellbeing, University of Glasgow, 1055 Great Western Road, \\ Glasgow, G12 0XH \\ Jill Holm-Denoma, Ph.D., Clinical Professor \\ Department of Psychology, Frontier Hall, Room 137, 2155 S. Race St. Denver, CO \\ 80208. \\ Tracy K. Witte, Ph.D., Professor \\ 226 Thach Hall, Auburn University, Auburn, AL 36849 \\ John Ruscio, Ph.D., Professor \\ Department of Psychology, The College of New Jersey, Ewing, NJ 08628.
}

Correspondence: Andy P. Siddaway, Suicidal Behaviour Research Laboratory, Institute of Health \& Wellbeing, University of Glasgow, 1055 Great Western Road, Glasgow, G12 0XH.

Email: Andy.Siddaway@glasgow.ac.uk 


\begin{abstract}
A central question in psychological science concerns whether psychological constructs are best conceptualized as dimensional or consist of one or more categories. The present study uses contemporary taxometric procedures to examine the latent structure of suicidal thoughts, with implications for how suicidal thoughts and behavior (STB) ought to be conceptualized, assessed, measured, and managed. Three nonredundant taxometric procedures (MAMBAC, MAXEIG, and L-Mode) were performed on various sets of indicators, and analyses were replicated across two large samples that included large numbers of individuals reporting current and recent STB. Results provide further evidence that the latent structure of suicidal thoughts is best understood as dimensional. However, inconsistent findings across studies and the relatively small number of taxometric studies conducted to date both suggest that it is premature to draw clear or definitive conclusions about the latent structure of STB being dimensional or categorical based on taxometric evidence. We report a metaanalysis of the current literature which evidences this ambiguity. We provide a detailed, critical discussion of the STB taxometric literature and outline key directions for future taxometric studies in this area, particularly how taxometric analysis relates to testing 'ideation to action' theoretical models, which hypothesize that the development of suicidal ideation and the progression from suicide desire to attempting suicide are distinct processes with distinct explanations/mechanisms. It remains entirely possible that qualitatively distinct types of STB (e.g., representing ideation vs. action) or populations have different latent structures indicating different levels of risk.
\end{abstract}




\section{Public Significance Statement}

This study provides further evidence that the latent structure of suicidal thoughts is dimensional. However, overall, the current taxometric evidence-base is relatively small and presents contradictory results regarding the latent structure of suicidal thoughts and behaviour (STB). Important directions for future taxometric research on STB are outlined to help reach a clear overall picture.

Keywords: suicide, injury, taxometric analysis, latent structure, conceptualization 


\section{Re-Examining the Latent Structure of Suicidal Thoughts Using \\ Taxometric Analysis: Implications for Testing Ideation to Action Theoretical Models of Suicidal Thoughts and Behavior}

A central and ongoing debate in psychological science concerns whether psychological constructs are best conceptualized as dimensional or consist of one or more categories. Dimensions refer to attributes that all people possess in some quantitative degree (e.g., neuroticism), whereas categories, types, or classes refer to attributes by which individuals differ qualitatively (e.g., pregnancy). Understanding and characterizing whether individual differences are best understood in terms of quantitative differences in degree or qualitative differences in kind has critical implications for how to conceptualize and measure mental health problems, including (1) explaining differences and boundaries between normality and abnormality and between psychological difficulties and well-being (Siddaway et al., 2018; Siddaway et al., 2017); and (2) establishing whether categorical psychiatric diagnoses or empirically derived dimensions provide a more valid and reliable classification approach (Clark et al., 2017; Conway et al., 2019; Markon et al., 2011; Regier et al., 2013).

Various methodological approaches have been used to explore and test the latent structure of psychological constructs. Taxometric analysis is generally accepted as a key methodology in this respect, and Monte Carlo studies have consistently demonstrated that taxometric procedures are capable of identifying taxa, if they exist, with a high degree of accuracy (Ruscio et al., 2010). A taxon is defined as a nonarbitrary latent class; taxon membership is discrete and either/or, with an empiricallyderived boundary separating members and non-members. Taxometric methods do not assume a specific latent structure and use multiple, mathematically non-redundant 
procedures to provide distinctive and valid information to compare taxonic and dimensional models (Ruscio et al., 2006, 2010). Additionally, taxometric methods are agnostic regarding the origin of different classes, which could be biological in nature (e.g., pregnant women are categorically distinct from women who are not pregnant), but need not be (e.g., nurses are categorically distinct from doctors). Importantly, this suite of techniques can be used to investigate latent structure regardless of whether observed variation is continuous or categorical. Finding that a psychological construct has a categorical latent structure does not mean that no meaningful quantitative variation exists among group members as there can be dimensional variation within categories (Ruscio et al., 2006) - i.e. differences of kind as well as degree. The research question that the taxometric method addresses is whether or not a categorical boundary exists that separates distinct taxon and complement groups.

The vast majority of the available evidence across different methodologies suggests that the latent structure of most clinical and non-clinical psychological constructs is dimensional (Clark et al., 2017; Conway et al., 2019; Haslam et al., 2020; Markon et al., 2011; Regier et al., 2013; Ruscio, 2019). This body of data is increasingly being used to argue for a paradigm shift in which psychiatric diagnostic categories are replaced by empirically derived dimensions (e.g., Hierarchical Taxonomy of Psychopathology (HiTOP; Kotov et al., 2017); National Institute of Mental Health's Research Domain Criteria (RDoC) framework; Cuthbert \& Kozak, 2013). When evidence of taxonicity has been found, it has often been a matter of much debate and contradictory evidence (Ruscio, 2019), highlighting that taxometric results are subject to the same replication issues as other research designs, and pointing to the need for replication efforts to clarify the overall picture. The current study replicates previous taxometric analyses of the latent structure of suicidal 
thoughts in two large, independent samples, with implications for research, theory, measurement, and clinical practice.

\section{Importance of Clarifying the Latent Structure of Suicidal Thoughts and}

\section{Behavior}

Suicide is a leading cause of death worldwide (World Health Organization, 2018) and a global public health priority. Globally, about 800,000 people die by suicide each year, which is more than in all armed conflicts and natural disasters combined (World Health Organization, 2018). These suicide deaths are in addition to an estimated 25 million annual suicide attempts (Crosby et al., 2011) and approximately 140 million annual suicide ideators worldwide (Borges et al., 2008). Establishing the latent structure of suicidal thoughts and behaviour (STB) is critical because, at present, it is not clear why some people develop suicidal thoughts or what explains the transition from thoughts to behaviour, and the conceptualization of STB in relation to nonsuicidal self-injury (NSSI) remains a matter of ongoing debate (Siddaway et al., 2019a). Clarifying how best to conceptualize and measure STB has the potential to improve research and practice because describing and explaining are prerequisites to effective identification, prediction, and interventions; however, the complex phenomenology of STB is difficult to explain.

Although STB are generally understood as a thought-behavior continuum that ranges from the absence of suicidal thoughts, to the presence of suicidal thoughts, to non-fatal suicidal behavior (making a suicide attempt), to fatal suicidal behaviour, this is a rational rather than empirically based conceptualization. In fact, there are empirical and theoretical reasons to suspect that the latent structure of STB might be best understood as categorical rather than continuous. For instance, several ecological momentary assessment studies have demonstrated that suicidal ideation is highly 
transient and dynamic, increasing and decreasing considerably over the course of most days (Kleiman et al., 2017; Nock et al., 2009). These findings suggest that individuals who are suicidal and/or make a suicide attempt enter a state of mind that is qualitatively or quantitatively different relative to their usual psychology and functioning, which may reflect (but is not necessarily indicative of) a categorical latent structure. Increasing recognition of the transient and dynamic nature of STB has led researchers to propose two new psychiatric diagnoses - the Suicide Crisis Syndrome (Galynker et al., 2016) and Acute Suicidal Affective Disturbance (Stanley et al., 2016) - for inclusion in the next revision of the Diagnostic and Statistical Manual of Mental Disorders to explicitly account for acute, drastic spikes in STB. Additionally, contemporary STB theoretical models (so-called 'ideation to action' frameworks; see Klonsky et al., 2017) explicitly distinguish between suicidal thoughts and suicidal behavior, implying a categorical difference, and the field is increasingly seeking to clarify what explains the transition from considering and desiring suicide to attempting suicide.

Pending further study, evidence for a STB taxon could support a state and/or a trait-based conceptualization of STB in that a STB taxon might represent a particular subpopulation and/or a "tipping point" that characterizes the emergence of suicidal thoughts or the transition from ideation to action (i.e. a categorically distinct outcome occurring when a latent dimensional influence exceeds a critical value). As articulated by Bryan (2019), evidence in favor of the latter explanation would be characteristic of a "cusp catastrophe model," in which sudden transitions from one state to another occur as a consequence of comparatively smaller change processes in other variables (Zeeman, 1976). 
Identifying a STB taxon would have important implications for research methodology and clinical practice because taxon members and non-members could differ substantially in risk or clinical presentation and therefore require different assessment or management approaches. Existing efforts to stratify people into high/low risk groups have demonstrated unusably poor sensitivity and specificity (e.g., because almost half of all the people who die by suicide come from the 'low' risk strata; Large et al., 2017). Identifying an empirically valid structure of STB might, therefore, have the potential to improve predictive accuracy. If a suicide taxon is robustly and reliably found, measures of the taxon (developed in subsequent research) could be combined with other information to more efficiently and accurately identify clinical needs and risks.

Equally, clarifying the absence of a STB taxon (if this is the case) would be valuable because artificially reducing continuous constructs into arbitrary dichotomies reduces measurement precision and statistical power (Cohen, 1983; MacCallum et al.,2002), losing meaningful variability which could decrease accuracy in clinical risk assessments and research designs and when trying to map trajectories of change. A meta-analysis of assessments of psychopathology constructs found that dimensional measures were, on average, $15 \%$ more reliable and $37 \%$ more valid than categorical measures, regardless of the clinical status of the sample or the type of psychopathology (Markon et al., 2011). Dimensional results would also have key implications for measurement because the goal of measurement of dimensional variables is to capture individual differences throughout the full range of variation along the construct, whereas for categorical variables the goal is to differentiate taxon members from non-members at the category boundary.

\section{Previous Taxometric Analyses of Suicidal Thoughts and Behavior}


Four taxometric studies of STBs have been published to date. These studies have examined different collections of STB indicators ${ }^{1}$ and found varying results, raising the possibility that the authors have not necessarily examined the latent structure of the same construct. Liu et al. (2015) conducted a taxometric analysis using a sample of 334 American adolescents presenting with clinically elevated depression symptoms. Three potential taxon indicators of suicidal ideation (morbid, passive, and active suicidal ideation) were derived and results from two taxometric procedures supported a dimensional structure (mean comparison curve fit index $[\mathrm{CCFI}]=0.38$; guidelines for interpreting CCFI values is provided below) .

Unfortunately, interpretation of these findings is potentially complicated because the authors did not report the results of the Mean Above Minus Below A Cut (MAMBAC; Meehl \& Yonce, 1994) taxometric procedure, despite reporting results from the MAXimum EIGenvalue (MAXEIG; Waller \& Meehl, 1998) and Latent Mode factor analysis (L-Mode; Waller \& Meehl, 1998) procedures.

Orlando et al. (2015) conducted a taxometric analysis using 1,525 American female college students with a history of intentional self-injury, irrespective of suicidal intent. The authors used five indicators of STB - suicidal intent; lifetime history of attempting suicide, including specific planning; lifetime frequency of intentional self-injury (irrespective of suicidal intent); lifetime number of methods used to self-injure (irrespective of suicidal intent); lifetime severity of intentional selfinjury (irrespective of suicidal intent) - and also found evidence for a dimensional structure $($ mean CCFI $=0.32)$.

\footnotetext{
${ }^{1}$ We describe taxometric indicators very carefully due to the critical need to be precise when interpreting the nature of the available evidence (see Discussion section). Our taxometric indicator descriptions are based on item or measure content (i.e. what was measured) and occasionally differ from the labels used by authors of the original studies.
} 
In contrast, the two more recent taxometric analyses found that STB have a categorical latent structure. Witte et al. (2017) conducted a taxometric analysis of STB using a sample of 1,773 Americans who were predominantly military personnel. Their indicators included current suicidal ideation and planning, current specific planning for a suicide attempt, lifetime worst-point suicidal planning and behavior, lifetime number of suicide attempts, objective lethality of most serious suicide attempt, and current insomnia. Results showed clear evidence of a taxonic structure $($ mean CCFI $=0.75)$.

In a sample of 2,385 American inpatient adults residing at a privately-funded psychiatric hospital, Rufino et al. (2018) also found evidence in support of taxonicity (mean CCFI $=0.68)$. Rufino et al. (2018) used four indicators that were the same as those used by Witte et al. (2017) (current specific planning for a suicide attempt, current suicidal desire and ideation, lifetime number of suicide attempts, objective lethality of most serious suicide attempt) and one indicator not used by Witte et al. (2017; lifetime specific planning for a suicide attempt); whilst Witte et al. (2017) included two indicators that were not used by Rufino et al. (2018) (lifetime worstpoint suicidal planning and behavior, current insomnia).

\section{The Current Study}

Recent years have drawn attention to the need to increase the reproducibility of psychological science and engage in replication attempts (see Siddaway et al., 2019b; Tackett et al., 2019). The purpose of the current study is to re-examine the latent structure of suicidal thoughts to determine whether it is dimensional or categorical. To do so, we use contemporary taxometric procedures to conduct conceptual replications of the small body of previous research in which taxometric 
analysis has been used to explore the latent structure of STB. We replicate our analyses twice, across two large samples.

\section{Method}

\section{Participants and Procedure}

Taxometric indicators were derived from two datasets collected during a large scale development and validation project in relation to the Suicide Attempt Beliefs Scale (SABS) and the Nonsuicidal Self-Injury Beliefs Scale (NSIBS; see Siddaway et al., 2019a) ${ }^{2}$. These datasets were selected a priori based on their suitability for answering the research question using taxometric analysis. Some participants $(N=47$ [8\%] of Sample 1; $N=62$ [10\%] of Sample 2) were excluded due to small amounts of incomplete taxometric indicator data, resulting in $N=603$ in Sample 1 and $N=602$ in Sample 2. There were no statistically significant differences between included and excluded participants on demographic or STB variables. The final samples consisted of predominantly female, white participants, whose average age was approximately 28 years. Detailed demographic information and information on STB characteristics is presented in Table 1 and further details are presented in Siddaway et al. (2019a).

Participants were recruited online from a broad range of suicide, self-harm, and mental health forums, websites, and charities worldwide. This recruitment strategy was specifically adopted in order to obtain large samples that are heterogeneous with respect to SIB characteristics due to the low incidence of STB (Siddaway et al.,2020) and evidence that: (1) individuals reporting STB present to physical and mental health services (NCISH, 2018); (2) most ( 70\%) STB episodes do not result in presentation to clinical services (Hawton et al., 2009); (3) STB is highly stigmatized and often secretive (NICE, 2011); and (4) most ( 70\%) people

\footnotetext{
${ }^{2}$ Samples 1 and 2 are, respectively, 'Sample 6' and 'Sample 5' from the previously described scale development/validation project (Siddaway et al., 2019a).
} 
who die by suicide have not been in contact with mental health services in the 12 months before death (NCISH, 2018). Participants were unpaid. Ethical approval was granted prior to data collection; participants provided informed consent for their data to be used for secondary analyses. Online participation also enabled the order of measure administration to be randomized, thereby eliminating an important potential source of error (Siddaway et al., 2019a).

\section{Measures: Taxometric Indicators}

The two existing datasets contained several theoretically and empirically relevant measures that could potentially be used as indicators for our taxometric analyses. Many of the potential indicators were apparent in both samples. Taxometric indicators were identified on an empirical basis by evaluating whether each potential indicator had the ability to validly distinguish latent classes (i.e., Cohen's $d \geq 1.25$ and within-class correlations $\leq .30$; Meehl, 1995; more details on this process are provided below). Indicator validity is a key prerequisite for conducting robust taxometric analyses.

Various potential indicators were considered (see Supplementary material for indicators that were not retained). Indicators that failed to meet data requirements for taxometric analysis were eliminated in an iterative fashion. In Sample 1, seven potential indicators were considered in the first round of validity analyses; the indicators which did not meet data requirements for taxometric analysis in that round were dropped; the process was then repeated until a pool of indicators remained that all met the criteria described above. This process led to the identification of several suitable taxometric indicators, which are described further below. Indicators were identified in Sample 2 using the same procedures, beginning with 10 potential indicators (see Supplementary material for indicators that were not retained). This 
process ultimately eliminated indicators measuring suicidal behavior. Similar indicators were identified in both samples. The final set of indicators for both samples and their psychometric properties are described in more detail next.

\section{Sample 1.}

Current suicidal ideation and planning. This indicator was constructed from the Depressive Symptom Inventory-Suicidality Subscale (DSI-SS; Metalsky \& Joiner, 1997), a 4-item self-report questionnaire designed to identify the frequency and intensity of suicidal ideation and impulses in the past two weeks, rated on a 4-point scale. Similar to Witte et al. (2017), we initially excluded item 2, as this item reflects specific planning for an attempt, whereas the other three items reflect more general suicidal desire and ideation; however, because the validity analyses demonstrated problematic nuisance correlations among the two DSI-SS variables (i.e., DSI-SS item 2 and DSI-SS items 1, 3, and 4), we chose to combine all four items into a composite indicator. The DSI-SS has been shown to have reasonable psychometric properties (Joiner et al.,2002). The four-item DSI-SS had an $\alpha$ of .89 .

Duration of suicidal thoughts. Participants were asked: When you think about killing yourself (attempting suicide), how long do those thoughts typically last? This was measured on a $0-11$ scale $(0=$ never have thought about suicide, $1=$ under 5 minutes, $2=5-15$ minutes, $3=15-30$ minutes, $4=30-45$ minutes, $5=45-60$ minutes, $6=1-2$ hours, $7=2-3$ hours, $8=3-4$ hours, $9=4-5$ hours, $10=5$ hours to all day, 11 $=$ multiple days).

\section{The belief that attempting suicide is the only option for solving one's}

problems. Participants rated their endorsement of the following belief using a 7-point Likert scale ranging from Strongly disagree (1) to Strongly agree (7): Attempting suicide is the only option I have for solving my problems. This item was drawn from 
the Dependence subscale of the recently developed SABS (Siddaway et al., 2019a).

This subscale measures perceptions that attempting suicide is the only option for selfregulation; that there are no alternative options; that attempting suicide provides important advantages; and a perception that suicide attempts are not necessarily/always expected to result in death.

\section{Sample 2.}

\section{Current suicidal desire and ideation, perceived capability to make a suicide}

attempt, and suicide attempt plans and preparations. This indicator was constructed from the total score of the Beck Scale for Suicide Ideation (BSS: Beck \& Steer, 1991). The BSS consists of 21 items. The two optional items (20 and 21) were not administered. The BSS has strong psychometric properties (Beck \& Steer, 1991). Factor analytic results generally support a two-factor solution: Suicidal Desire and Ideation, which measures a desire for death, frequency of suicidal ideation, and lacking deterrents for suicide; Resolved Plans and Preparations which measures specific plans and suicidal intent. However, there is no consensus on the composition of the two BSS factors (Siddaway et al., 2019a). We used the BSS total score as an indicator because the resolved plans and preparations factor we derived (i.e. items 12 , $13,14,16,17$, and 18$)$ had inadequate internal consistency $(\alpha=.75)$. The BSS has three response options for each item. Readers are asked to select which option best describes how they have been feeling over the past week. Internal consistency was $\operatorname{good}(\alpha=.92)$.

Duration of suicidal thoughts. See description provided for Sample 1.

The belief that attempting suicide is the only option for solving one's problems. See description provided for Sample 1.

\section{Data Analytic Strategy}


Three nonredundant taxometric procedures were used to address the types vs. dimensions question: MAMBAC (Meehl \& Yonce, 1994), MAXEIG (Waller \& Meehl, 1998), and L-Mode (Waller \& Meehl, 1998). MAMBAC (Meehl \& Yonce, 1994) generates a sliding "cut" for an "input" indicator and then computes mean differences for cases falling above and below each sliding cut on the score distribution of an "output" indicator. A graph of mean difference scores is plotted; relatively flat or concave curves suggest dimensional structure, whereas relatively peaked or convex curves suggest taxonic structure. MAXEIG (Waller \& Meehl, 1998) generates a curve showing how strongly two or more "output" indicators are associated within a series of ordered subsamples of cases along an "input" indicator. A graph of eigenvalues is plotted; relatively flat curves suggest dimensional structure, whereas relatively peaked curves suggest taxonic structure. L-Mode (Waller \& Meehl, 1998) conducts a factor analysis using at least three indicators. Factor scores are estimated for the first factor using Bartlett's (1937) weighted least squares method, and the density of these scores is plotted. A unimodel graph suggests dimensional structure, whereas a bimodal graph suggests taxonic structure.

The taxometric analyses were conducted using Ruscio and Wang's (2017) R program for taxometric analysis, which incorporates the latest empirically supported taxometric techniques (Ruscio et al., 2018). The default settings for each analysis (e.g., number of cuts, number of replications ${ }^{3}$ ) was used on the basis of findings from Monte Carlo research on how best to implement each procedure (Ruscio et al.,2011).

\footnotetext{
${ }^{3}$ Number of cuts refers to a component of MAMBAC analyses in which a series of equally spaced "cuts" are made between cases sorted on an input variable ("x"), and the mean of another variable ("y") is calculated separately both above and below each cut. At each increasing value of "x," the mean of " $y$ " below the cut is subtracted from the mean of " $y$ " above the cut. The resulting values at each cut are plotted. Number of replications refers to a procedure by which scores on the input indicator are randomly re-sorted to shuffle the order of cases with tied scores, and the analysis is re-run.
} 
Historically, researchers have evaluated the output of taxometric methods by studying the shape of taxometric curves. In-line with standard practice, we visually display relative model fit for each taxometric method (see Figures 1 and 2). Each averaged curve can be tentatively considered categorical, dimensional, or ambiguous on the basis of whether it most closely resembles the comparison categorical data plot, the comparison dimensional data plot, or equally well resembled both sets of comparison data plots.

Judgments about taxonicity were primarily based on the CCFI (Ruscio et al., 2007), which is generally considered to be the most robust and optimal interpretive metric and helps to reduce the subjectivity involved in performing taxometric analyses and interpreting results (Ruscio et al., 2007, 2010, 2018). The CCFI quantitatively determines the relative fit between one's data and the results for parallel analyses of artificial categorical and dimensional comparison data that reproduce the distributions and correlations observed in the actual data/sets. The CCFI can range from 0 to 1 , with values of 0 indicating a dimensional model and values of 1 indicating a categorical model. Ruscio et al. (2018) recommend that CCFI values falling between .45 and .55 should be interpreted with caution because they do not clearly indicate a categorical or dimensional structure (Ruscio et al., 2007, 2018). A CCFI $<.45$ is indicative of a dimensional structure and a CCFI $>.55$ is indicative of a categorical (i.e., taxonic) structure (Ruscio et al., 2018). Importantly, the CCFI can be computed separately on the basis of the output of MAXCOV, MAMBAC, and LMode analyses. Thus, when multiple taxometric procedures are used, the average CCFI across those procedures can be interpreted as a robust way of evaluating the evidence (Ruscio et al., 2010). 
Prior to performing the planned taxometric analyses, preliminary analyses were conducted to obtain taxon base rate estimates based on algorithms used by the $\mathrm{R}$ program (Ruscio et al., 2017). A preliminary series of MAMBAC and MAXEIG analyses was conducted solely to obtain estimates of the taxon base rate. Each MAMBAC curve provides a base-rate estimate via a formula based on the relative heights of the two endpoints of the curve; these estimates are then averaged to yield one overall MAMBAC base rate estimate. Each MAXEIG curve provides an estimate based on the location of the subsample corresponding to the largest data point on the curve, and once again these estimates are averaged. Finally, the base rate estimates from the MAMBAC and MAXEIG analyses are themselves averaged. The averaged base rate estimate from preliminary MAXEIG and MAMBAC analyses was .46 in Sample 1 and .47 in Sample 2, and these estimates were used to assign cases to putative taxon and complement groups in each sample. These putative groups were used, in turn, to check the adequacy of the data for the planned taxometric analyses (i.e., to ensure that the indicator variables yielded sufficiently large differences between putative groups and had tolerably low levels of within-class correlations) and to guide the generation of artificial categorical comparison data. Once cases had been assigned to groups, indicators were examined to ensure they could sufficiently differentiate between the two putative groups and had tolerably low levels of withinclass correlations. Indicators that met these criteria were used to generate the artificial categorical comparison data needed to calculate CCFI values (Ruscio et al., 2017).

The consistency and robustness of the taxometric findings for each sample was further examined using CCFI profiles (Ruscio et al., 2018). This procedure generates CCFI values when taxon base rates range from .025 to .975 in increments of .025. Cases are initially assigned to taxon and complement groups using each taxon 
base rate and this classification is then used to generate a new population of categorical comparison data. MAMBAC, MAXEIG, and L-Mode analyses are performed for random samples from this population and the CCFIs are recorded. This procedure is repeated for each taxon base rate and when all analyses are completed the CCFIs are plotted across taxon base rates to form a profile. CCFI profiles that indicate taxonicity demonstrate CCFI values that are predominantly or entirely above the .50 line, peaking near the location of an actual taxon base rate. CCFI profiles that indicate dimensionality demonstrate CCFI values that are predominantly or entirely below the .50 line, with no clear peak because there is no taxon whose base rate is being estimated. In addition to this visual inspection, Ruscio et al. (2018) provide empirical evidence that the average CCFI produced by a CCFI profile very effectively differentiates categorical and dimensional data.

\section{Results}

\section{Indicator Selection}

Sample 1. Indicator validity scores ranged from $d=1.26-1.80(M=1.53$; see Table 2) for the three indicators used in the taxometric analyses, and all surpassed the threshold suggested by Meehl (1995). The within-class correlations were all below $r$ $=.30$, indicating that indicators were not highly correlated within the putative taxon or complement groups (see Table 2). Finally, the mean base rate estimate (.46) surpassed the lower threshold established by Ruscio et al. (2010), indicating that putative taxa were sufficiently represented in each dataset.

Sample 2. Indicator validity scores ranged from $d=1.20-2.66(M=1.73$; see Table 2) for the three indicators used in the taxometric analyses, and all but one surpassed the threshold suggested by Meehl (1995); we retained the indicator with slightly poorer validity than recommended by Meehl because its other psychometric 
properties were strong. The within-class correlations were all well below $r=.30$ (see Table 2), indicating that indicators were not highly correlated within the putative taxon or complement groups (see Table 2), and the mean base rate estimate (.47) surpassed the lower limit threshold established by Ruscio et al. (2010), indicating that putative taxa were sufficiently represented in each dataset.

\section{Taxometric Analyses}

Sample 1. The CCFI values for the each of the taxometric procedures are as follows: $\mathrm{MAMBAC}=0.34, \mathrm{MAXEIG}=0.50, \mathrm{~L}-\mathrm{Mode}=0.42$. The average of the CCFIs across the three taxometric procedures (0.42) was most similar to that expected under a dimensional model as opposed to a categorical one (i.e., CCFI < 0.45 ). In addition, the taxometric graphs for each procedure are most consistent with a dimensional interpretation (see Figure 1). Figure 2 presents the CCFI profile for Sample 1. Almost every data point is below .50, and the mean CCFI value of the averaged profile $(0.42)$ indicates dimensionality.

Sample 2. The CCFI values for the each of the taxometric procedures are as follows: $\mathrm{MAMBAC}=0.29, \mathrm{MAXEIG}=0.38, \mathrm{~L}-\mathrm{Mode}=0.27$. The results for Sample 2 were similar to those for Sample 1 and again were indicative of an underlying dimension rather than underlying categories. The mean CCFI value (0.32) supported a dimensional latent structure. Again, the taxometric graphs for each procedure are most consistent with a dimensional interpretation (see Figure 3). Figure 4 presents CCFI profiles for Sample 2. Almost every data point is below .50 and the mean CCFI value of the averaged profile is 0.34 , which clearly indicates dimensional data.

\section{Discussion}

Determining whether psychological constructs are best understood as dimensional or categorical is a central question in psychological science and has 
direct implications for how individual differences in STB ought to be conceptualized, assessed, measured, and managed in treatment. Across two relatively large samples, we found evidence that the latent structure of suicidal thoughts is best understood as continuous. A key strength of the present study is that our two community-based samples were heterogeneous with respect to STB experience and included large numbers of individuals reporting current and recent STB; features that are important, given the low incidence of STB (Siddaway et al., 2020). The high proportion of STB experiences in our samples means that we are likely to have sampled individuals from each of the 'ideation' and 'action' conditions purported by recent theoretical models (see Klonsky et al., 2017), which is important because taxometric analysis can only identify separate classes of individuals when there is sufficient heterogeneity in type and severity of symptoms.

When interpreting the present findings, it is important to keep in mind that taxometric analysis clarifies latent structure. Concluding that a particular psychological construct is dimensional does not suggest that that variable is the result of simple additive influences, just that the influences that bear on it do not generate latent discontinuities. Moreover, although our findings support the dimensional nature of suicidal thoughts, they do not rule out the possibility that suicidal thoughts may interact with other psychopathology variables to produce qualitatively distinct clinical profiles. Whilst clinicians and researchers often refer to (arbitrary) groups using categorical descriptors (e.g., "depressed," "suicidal"), this terminology is merely a convenient (and sometimes careless) way of demarcating rough regions on what are generally in reality quantitative traits, dimensions, or factors (Meehl, 1992). The existence and use of categorical terminology to describe and differentiate "normal" and "abnormal" (or clinical vs. non-clinical) psychological variation does not mean 
that the variables in question are in fact latent types; determining whether a variable is taxonic is an empirical question (Meehl, 1992), and our results suggest that suicidal thoughts are not taxonic.

\section{Making Sense of Inconsistent STB Taxometric Results}

Including the present study, six taxometric studies of STB have been conducted to date and these provide contradictory results regarding the latent structure of STB. These studies: (i) possessed adequate sample sizes for robustly performing taxometric analyses; (ii) included indicators that met data requirements for taxometric analysis; (iii) used a sufficient number of taxometric indicators to robustly perform taxometric analyses (e.g., evidence indicates that latent structure can be accurately tested using only two taxometric indicators; Ruscio \& Walters, 2011); and (iv) have been conducted analyses in-line with best practice, apart from the omission of MAMBAC results by Liu et al. (2015).

The combination of inconsistent findings and the relatively small number of taxometric studies both suggest that it may currently be premature to draw clear or definitive conclusions about the latent structure of STB being dimensional or categorical based on taxometric evidence. In an attempt to bring clarity to the disparate findings, we conducted a meta-analysis of the taxometric results in this area, including our own, following the approach employed by Haslam et al. (2020). This revealed a mean CCFI of 0.48 (95\% CI 0.33 - 0.64), with no significant betweenstudy heterogeneity $(p=.18)$. As discussed, the literature recommends that CCFI values falling between .45 and .55 are ambiguous and do not clearly indicate a categorical or dimensional structure (Ruscio et al., 2007, 2018). These meta-analytic results should also be interpreted with caution given that the proportionality constant will not be estimated accurately based on just six studies. Overall, these results 
demonstrate empirically that it is currently premature to draw clear or definitive conclusions about the latent structure of STB being dimensional or categorical based on taxometric evidence, and they provide less detail and context than a narrative discussion of the current evidence base.

Taking into account the extent and nature of the available evidence, there seem to be two potential explanations for the STB taxometric findings obtained to date. Either the current taxometric evidence base consists of (1) studies with genuinely different findings regarding the same latent construct (STB), as often happens with replication attempts (see Siddaway et al., 2019b); or (2) some studies that measure a particular latent STB construct (e.g., suicidal thoughts) and some studies that measure a different latent STB construct (e.g., suicidal thoughts and behavior). 'Ideation to action' theoretical models - which suggest that the development of suicidal ideation and the progression from suicide desire to attempting suicide are distinct processes with distinct explanations/mechanisms imply that the second explanation is true.

Careful inspection of the samples and indicators used in the different STB taxometric studies conducted to date reveals some potentially important differences between the studies that identified evidence of a STB taxon and those that did not. Although most of the indicators used have been very similar across studies, three indicators appeared in the two studies that found evidence for a taxonic structure (Rufino et al., 2018; Witte et al., 2017), but not in the studies which found evidence of a dimensional structure. These are: (1) lifetime worst point suicidal planning and behavior, (2) objective lethality of most serious suicide attempt, and (3) current insomnia (although insomnia was only included in Witte et al., 2017). These indicators could be viewed as indications of more serious or acute suicide risk. These 
indicators potentially characterize the transition from considering suicide to making a suicide attempt or the nature of suicidal behavior, whereas the indicators used in all studies potentially characterize the emergence of suicidal thoughts or the nature of suicidal thoughts. As stated, in the current study we considered various potential taxometric indicators for inclusion in each of our two samples and were forced to exclude indicators measuring suicidal behaviour because these failed to meet data requirements for taxometric analysis (see Supplementary material).

Some further differences can be noted between the studies that found evidence of a STB taxon compared to the studies that did not. First, the two studies that identified a STB taxon included a much higher proportion of men (48\% female in Rufino et al., 2018 and 26\% female in Witte et al., (2017); compared to 70\% female samples used by Liu et al., 2015 and Orlando et al., 2015; and 83\% female samples in the present study). Second, the two studies that identified a STB taxon were recruited from institutional settings (i.e. an inpatient psychiatric hospital and research participants enrolled in 16 different military studies, including non-treatment seeking individuals, inpatients, and outpatients), whereas the studies that found evidence of dimensionality recruited participants from community settings who were not necessarily treatment seeking. Third, the two studies that identified a STB taxon had slightly older samples ( $\mathrm{M}$ age $=34$ in both studies), whereas the studies that found evidence of dimensionality used adolescents (Liu et al., 2015; M age = 16 years), college students (Orlando et al., 2015; M age = 21 years), or community samples with a mean age of 28 (the present research). These sample differences raise the possibility that the latent structure of STB might be different in particular populations or recruitment settings, although we are not aware of any theoretical reason to make specific hypotheses or interpretations in this regard, and we note that a recent meta- 
analysis of taxometric research found that CCFI values did not significantly vary depending on publication year, sample size, or sample type (undergraduate, clinical, community; Haslam et al., 2020). Unfortunately, it is not possible to evaluate whether the differing results in this area to date could be attributed to systematic differences in STB characteristics across studies (e.g., number and recency of suicide attempts), as this information was not reported in all previous taxometric studies. The large numbers of individuals reporting current and recent STB in each of our samples challenges this possibility.

Taken together, the fact that varying results have been observed across studies that used different samples and indicators leaves open the possibility that different subtypes or facets of STB have different latent structures representing different subpopulations and/or that a "tipping point" (i.e. a categorically distinct outcome) characterizes the emergence of suicidal thoughts or the transition from ideation to action. The latent structure of suicidal behaviour enaction may ultimately prove to be taxonic whereas the latent structure of suicidal ideation and intention formation may be dimensional, as implied by 'ideation to action' theoretical models (see Klonsky et al., 2017). Unfortunately, it is beyond the scope of the present study to resolve the debate on whether the current taxometric evidence-base consists of studies with different findings on the same latent construct (STB) or an admixture of various STB constructs or different populations. As the current evidence-base indicates that STB is complex, multiply determined, and usually relatively episodic (Kleiman et al., 2017; Large et al., 2017; Nock et al., 2009; Siddaway et al., 2020), our own view is that STB is probably characterized by a complex latent structure wherein the broad construct of STB is dimensional and the narrower construct of serious suicidality is taxonic. 
Lastly, given the importance of thoughtful, accurate interpretation, it is noteworthy that the Orlando et al. (2015) study has been used by researchers and clinicians to support the notion that attempting suicide and NSSI form a single continuum or spectrum. However, a careful inspection of the indicators used by Orlando et al. (2015) reveals that this study did not include any indicators that explicitly measure only NSSI. The indicators used were: suicidal intent; lifetime history of attempting suicide, including specific planning; lifetime frequency of intentional self-injury (irrespective of suicidal intent); lifetime number of methods used to self-injure (irrespective of suicidal intent); and lifetime severity of intentional self-injury (irrespective of suicidal intent).

To conclude that the results presented by Orlando et al. (2015) indicate attempting suicide and NSSI form a single continuum appears to go beyond the data. It is analogous to purporting to test whether the latent structure of anxiety and depression symptoms form a single latent continuum using taxometric analyses, but including taxometric indicators that measure mixed depression and anxiety symptoms. The only way to use taxometric analysis to test whether the latent structure of anxiety and depression symptoms form a continuum is to include indicators that separately represent anxiety versus depression symptoms. This critical limitation to the Orlando et al. (2015) study means that the use of taxometric analysis to clarify whether attempting suicide and NSSI are distinct constructs or form a single continuum, remains unanswered. We are currently planning such a research study and note that at present, the evidence base indicates that STB and NSSI are both correlated, but independent constructs (see Siddaway et al., 2019a).

\section{Future Taxometric Research in This Area}


The various issues discussed clearly highlight the need for further taxometric research that includes (1) individuals representing the full spectrum of STB, so as to sample hypothesised subtypes of STB (e.g., individuals who have never experienced suicidal thoughts, individuals who have experienced suicidal thoughts and considered suicide but never made a suicide attempt, individuals who have made a suicide attempt, individuals who have made multiple suicide attempts); (2) indicators that explicitly sample variables representing both the ideation/motivational and action/volitional components of recent STB theoretical models. Including several indicators representing STB ideation and action and replicating findings across several samples would provide a robust test of the latent structure of the full spectrum of STB; and (3) large numbers of each gender, so taxometric analysis can be conducted using sub-samples of just men or women in case the latent structure of STB differs between those groups and could help to explain gender differences in death by suicide.

Further research is also warranted to determine whether results are generalizable to the general population, other populations (e.g., non-English speakers), and to non-self-report methods of assessing STB. Regarding generalizability, to facilitate interpretation and comparisons across studies it is important that future taxometric studies report STB sample composition characteristics (e.g., proportion of individuals who have attempted suicide, recency of suicide attempt). Regarding the method of data collection, some authors have speculated that self-report indicators may produce "pseudotaxonic" findings or otherwise potentially introduce noise into the taxometric output due to various response styles inherent in self-report assessments (Beauchaine \& Waters, 2003). The fact that a meta-analysis of taxometric research found no differences in rates of 
taxonic versus dimensional findings across self-report, observer-rated, and interviewbased data (Haslam et al., 2020) speaks to this potential concern, but it would still be helpful to address it empirically in this area.

If a body of evidence consistently emerges over time and methodologies which indicates the existence of one or more latent STB categories, it would be prudent to employ methodologies and analyses that allow both categories and dimensions to coexist. Factor mixture modeling could be especially relevant for this task because it classifies individuals into latent categories and allows for heterogeneity within latent groups (Muthen, 2006).

\section{Conclusion}

In closing, one of the long-standing, central questions facing psychological science concerns whether individual differences are best understood in terms of quantitative differences in degree or qualitative differences in kind. Inconsistent results and the nature of the studies that have been conducted to date mean that at this stage the jury is still out as to whether particular facets or subtypes of STB may have taxonic latent structures. Best practice guidelines for conducting systematic reviews (e.g., Siddaway et al., 2019b) highlight that because there are no established or agreed-upon criteria for deciding that a finding has replicated or what replication means, the emphasis should be on a consensus of findings across methodologies and statistical techniques that have matured to the stage where there is a clear overall picture. These are exciting possibilities for future studies to explore using taxometric analysis and other methodologies and analyses. 


\section{References}

Bartlett, M. S. (1937). The statistical conception of mental factors. British Journal of Psychology, 28, 97-104. https://doi.org/10.1111/j.2044-8295.1937.tb00863.x

Beauchaine, T. P., \& Waters, E. (2003). Pseudotaxonicity in MAMBAC and MAXCOV analyses of rating-scale data: Turning continua into classes by manipulating observer's expectations. Psychological Methods, 8, 3-15. https://doi.org/10.1037/1082-989x.8.1.3

Beck, A. T., \& Steer, R. A. (1991). Beck scale for suicidal ideation: Manual. New York, NY: Psychological Corporation.

Borges, G., Angst, J., Nock, M. K., Ruscio, A. M., \& Kessler, R. C. (2008). Risk factors for the incidence and persistence of suicide-related outcomes: A 10year follow-up study using the National Comorbidity Surveys. Journal of Affective Disorders, 105, 25-33. https://doi.org/10.1016/j.jad.2007.01.036

Bryan, C. (2019). A preliminary validation study of two ultra-brief measures of suicide risk: The suicide and perceived burdensomeness visual analog scales. Suicide and Life-Threatening Behavior, 49, 343-352. https://doi.org/10.1111/sltb.12447

Bryan, C, J., \& Harris, J. A. (2019). The structure of suicidal beliefs: A bifactor analysis of the suicide cognitions scale. Cognitive Therapy \& Research, 43, 335-344. https://doi.org/10.1007/s10608-018-9961-2

Clark, L. A., Cuthbert, B., Lewis-Fernandez, R., Narrow, W. E., \& Reed, G. M. (2017). Three Approaches to understanding and classifying mental disorder: ICD-11, DSM-5, and the National Institute of Mental Health's Research Domain Criteria (RDoC). Psychological Science in the Public Interest, 18, 72145. https://doi.org/10.1177/1529100617727266 
Cohen (1983). The cost of dichotomization. Applied Psychological Measurement, 7, 249 -253. https://doi.org/10.1177/014662168300700301

Conway, C, C., Forbes, M. K., Forbush, K. T., Fried et al., Hallquist, M., N., Kotov, R., Mullins-Sweatt, S. N., Shackman, A. J., Skodol, A E., South, S. C., Sunderland, M., Waszczuk, M. A., Zald, D. H., Afzali, M. H., Bornovalova, M. A., Carragher, N., Docherty, A. R., Jonas, K., G., Krueger, R. F., Patalay, P., Pincus, A. L., Tackett, J., L....Eaton, N. R. (2019). A hierarchical taxonomy of psychopathology can transform mental health research. Perspectives on Psychological Science, 14, 419-436. https://doi.org/10.31234/osf.io/wsygp

Crosby, A., Gfroerer, J., Han, B., Ortega, L., \& Parks, S. E. (2011). Suicidal thoughts and behaviors among adults aged 18 Years-United States, 2008-2009. Washington, DC: US Department of Health and Human Services, Centers for Disease Control and Prevention. https://doi.org/10.1037/e515442011-001

Cuthbert, B. N., \& Kozak, M. J. (2013). Constructing constructs for psychopathology: The NIMH research domain criteria. Journal of Abnormal Psychology, 122, 928-937. https://doi.org/10.1037/a0034572

Galynker, I. Yaseen, Z. S., Cohen, A., Benhamou, O., Hawes, M., Briggs, J. (2016). Prediction of suicidal behavior in high risk psychiatric patients using an assessment of acute suicidal state: The suicide crisis inventory. Depression \& Anxiety. 34, 147-158. https://doi.org/10.1002/da.22559

Haslam, N., McGrath, M. J., Viechtbauer, W., \& Kuppens, P. (2020). Dimensions over categories: A meta-analysis of taxometric research. Psychological Medicine, 50, 1418-1432. https://doi.org/10.1017/s003329172000183x 
Hawton, K., Rodham, K., Evans, E., \& Harriss, L. (2009). Adolescents who selfharm: A comparison of those who go to hospital and those who do not. Child and Adolescent Mental Health, 14, 24-30. https://doi.org/10.1111/j.14753588.2008.00485.x

Joiner, T. E., Pfaff, J. J., \& Acres, J. G. (2002). A brief screening tool for suicidal symptoms in adolescents and young adults in general health settings: reliability and validity data from the Australian National General Practice Youth Suicide Prevention Project. Behaviour Research and Therapy, 40, 47181. https://doi.org/10.1016/s0005-7967(01)00017-1

Kleiman, E.M., Turner, B.J., Fedor, S., Beale, E.E., Huffman, J.C., \& Nock, M.K. (2017). Examination of real-time fluctuations in suicidal ideation and its risk factors: Results from two ecological momentary assessment studies. Journal of Abnormal Psychology, 126, 726-738. https://doi.org/10.1037/abn0000273

Klonsky, E.D., Saffer, B.Y., \& Bryan, C.J. (2018). Ideation-to-action theories of suicide: A conceptual and empirical update. Current Opinion in Psychology, 22, 38-43. https://doi.org/10.1016/j.copsyc.2017.07.020

Kotov, R., Krueger, R. F., Watson, D., Achenbach, T. M., Althoff, R. R., Bagby, R. M.,.. \& Zimmerman, M. (2017). The Hierarchical Taxonomy of Psychopathology (HiTOP): A dimensional alternative to traditional nosologies. Journal of Abnormal Psychology, 126, 454-477. https://doi.org/10.31234/osf.io/zaadn

Large, M. M., Ryan, C. J., Carter, C., \& Kapur, N. (2017). Can we usefully stratify patients according to suicide risk? British Medical Journal, 359, j4627. https://doi.org/10.1136/bmj.j4627 
Liu, R. T., Jones, R. N., \& Spirito, A. (2015). Is adolescent suicidal ideation continuous or categorical? A taxometric analysis. Journal of Abnormal Child Psychology, 43, 1459-1466. https://doi.org/10.1007/s10802-015-0022-y

MacCallum, R. C., Zhang, S., Preacher, K. J., \& Rucker, D. D. (2002). On the practice of dichotomization of quantitative variables. Psychological Methods, 7, 19-40. https://doi.org/10.1037/1082-989x.7.1.19

Markon, K. E., Chmielewski, M., \& Miller, C. J. (2011). The reliability and validity of discrete and continuous measures of psychopathology: A quantitative review. Psychological Bulletin, 137, 856-879. https://doi.org/10.1037/a0023678

Meehl, P. E. (1992). Factors and taxa, traits and types, differences of degree and differences in kind. Journal of Personality, 60, 117-174. https://doi.org/10.1111/j.1467-6494.1992.tb00269.x

Meehl, P. E. (1995). Bootstraps taxometrics: Solving the classification problem in psychopathology. American Psychologist, 50, 266-275. https://doi.org/10.1037/0003-066x.50.4.266

Meehl, P. E, \& Yonce, L. J. (1994). Taxometric analysis: I. Detecting taxonicity using two quantitative indicators using means above and below a sliding cut (MAMBAC procedure). Psychological Reports, 74, 1059-1274. https://doi.org/10.2466/pr0.1996.78.3c.1091

Metalsky, G. I., \& Joiner, T. E. (1997). The hopelessness depression symptom questionnaire. Cognitive Therapy and Research, 21, 359-384. https://doi.org/10.1037/t59143-000 
Muthén B. (2006). Should substance use disorders be considered as categorical or dimensional? Addiction, 101, 6-16. https://doi.org/10.1111/j.13600443.2006.01583.x

National Institute for Health and Care Excellence (2011). Self-harm: Longer-term management. London: NICE.

Nock, M. K., Prinstein, M. J., \& Sterba, S. K. (2009). Revealing the form and function of self-injurious thoughts and behaviors: A real-time ecological assessment study among adolescents and young adults. Journal of Abnormal Psychology, 118, 816-827. https://doi.org/10.1037/2152-0828.1.s.36

Orlando, C. M., Broman-Fulks, J.J., Whitlock, J. L., Curtin, L., \& Michael, K. D. (2015). Nonsuicidal self-injury and suicidal self-injury: A taxometric investigation. Behavior Therapy, 46, 824-33. https://doi.org/10.1016/j.beth.2015.01.002

Regier, D. A., Kuhl, E. A., \& Kupfer, D. J. (2013). The DSM-5: Classification and criteria changes. World Psychiatry, 12, 92-98. https://doi.org/10.1002/wps.20050

Rudd, M. D., Schmitz, W., McClenen, R., Joiner, T., Elkins, G., \& Claassen, C. (in preparation). The Suicide Cognitions Scale: A suicide-specific measure of hopelessness. https://doi.org/10.1037/t54628-000

Rufino, K. A., Marcus, D. K., Ellis, T. E., \& Boccaccini, M. T. (2018). Further evidence that suicide risk is categorical: A taxometric analysis of data from an inpatient sample. Psychological Assessment, 30, 1541-1547. https://doi.org/10.1037/pas0000613 
Ruscio, A. M. (2019). Normal versus pathological mood: implications for diagnosis. Annual Review of Clinical Psychology, 15, 179-205. https://doi.org/10.1146/annurev-clinpsy-050718-095644

Ruscio, J., Carney, L. M., Dever, L., Pliskin, M., \& Wang, S. B. (2018). Using the Comparison Curve Fix Index (CCFI) in taxometric analyses: Averaging curves, standard errors, and CCFI profiles. Psychological Assessment, 30, 744754. https://doi.org/10.1037/pas0000522

Ruscio, J., Haslam, N., \& Ruscio, A. M. (2006). Introduction to the taxometric method: A practical guide. Mahwah, NJ: Lawrence Erlbaum. https://doi.org/10.4324/9780203726549

Ruscio, J., \& Ruscio, A. M. (2008). Categories and dimensions: Advancing psychological science through the study of latent structure. Current Directions in Psychological Science, 17, 203-207. https://doi.org/10.1111/j.14678721.2008.00575.x

Ruscio, J., Ruscio, A. M., \& Carney, L. M. (2011). Performing taxometric analyses to distinguish categorical and dimensional variables. Journal of Experimental Psychopathology, 2, 170-196. https://doi.org/10.5127/jep.010910

Ruscio, J., Ruscio, A. M., \& Meron, M. (2007). Applying the bootstrap to taxometric analysis: Generating empirical sampling distributions to help interpret results. Multivariate Behavioral Research, 42, 349-386. https://doi.org/10.1080/00273170701360795

Ruscio, J., \& Walters, G. D. (2011). Differentiating categorical and dimensional data with taxometric analysis: Are two variables better than none? Psychological Assessment, 23, 287-299. https://doi.org/10.1037/a0022054 
Ruscio, J., Walters, G. D., Marcus, D. K., Kaczetow, W. (2010). Comparing the relative fit of categorical and dimensional latent variable models using consistency tests. Psychological Assessment, 22, 5-21. https://doi.org/10.1037/a0018259

Ruscio, J., \& Wang, S. B. (2017). RTaxometrics: Taxometric analysis. R package version 2.3. Available at https://CRAN.R-project.org/package=RTaxometrics.

Siddaway, A. P., Quinlivan, L., Kapur, N., O’Connor, R. C., \& de Beurs, D. (2020). Cautions, concerns, and future directions for using machine learning in relation to mental health problems and clinical and forensic risks: A brief comment on "Model complexity improves the prediction of nonsuicidal selfinjury” (Fox et el., 2019). Journal of Consulting and Clinical Psychology, 88, 384-387. https://doi.org/10.1037/ccp0000485

Siddaway, A. P., Taylor, P. J., \& Wood, A. M. (2018). Re-conceptualizing anxiety as a continuum that ranges from high calmness to high anxiety: The joint importance of reducing distress and increasing well-being. Journal of Personality and Social Psychology, 114, e1-e11. https://doi.org/10.1037/pspp0000128

Siddaway, A. P., Wood, A. M., \& Taylor, P. J. (2017). The Centre for Epidemiologic Studies-Depression (CES-D) scale measures a continuum from well-being to depression: Testing two key predictions of Positive Clinical Psychology. Journal of Affective Disorders, 213, 180-186. https://doi.org/10.1016/j.jad.2017.02.015

Siddaway, A. P., Wood, A. M., \& Hedges, L. V. (2019b. How to do a systematic review: A best practice guide to conducting and reporting narrative reviews, 
meta-analyses, and meta-syntheses. Annual Review of Psychology, 70, 747770. https://doi.org/10.1146/annurev-psych-010418-102803

Siddaway, A. P., Wood, A. M., O’Carroll, R. E., \& O’Connor, R. C. (2019a). Characterizing self-injurious cognitions: Development and validation of the Suicide Attempt Beliefs Scale (SABS) and the Nonsuicidal Self-Injury Beliefs Scale (NSIBS). Psychological Assessment, 31, 592-608. https://doi.org/10.1037/pas0000684

Stanley, I. H., Rufino, K. A., Rogers, M. L., Ellis, T. E., \& Joiner, T. E. (2016). Acute Suicidal Affective Disturbance (ASAD): A confirmatory factor analysis with 1442 psychiatric inpatients. Journal of Psychiatric Research, 80, 97-104. https://doi.org/10.1016/j.jpsychires.2016.06.012

Tackett, J. L., Brandes, C. M., King, K. M., \& Markon, K. E. (2019). Psychology's replication crisis and clinical psychological science. Annual Review of Clinical Psychology, 15, 579-604. https://doi.org/10.1146/annurev-clinpsy-050718095710

The National Confidential Inquiry into Suicide and Safety in Mental Health. (2018) Annual Report: England, Northern Ireland, Scotland and Wales. University of Manchester.

Van Orden, K. A., Cukrowicz, K. C., Witte, T. K., \& Joiner, T. E. (2012). Thwarted belongingness and perceived burdensomeness: Construct validity and psychometric properties of the Interpersonal Needs Questionnaire. Psychological Assessment, 24, 197-215. https://doi.org/10.1080/07481187.2018.1541938

Waller, N. G., \& Meehl, P. E. (1998). Multivariate taxometric procedures: Distinguishing types from continua. Newbury Park, CA: Sage. 
Witte, T. K., Holm-Denoma, J. M., Zuromski, K. L., Gauthier, J. M., \& Ruscio, J. (2017). Individuals at high risk for suicide are categorically distinct from those at low risk. Psychological Assessment, 29, 382-393. https://doi.org/10.1037/pas0000349

World Health Organization (2018, August 24). Suicide. Retrieved from https://www.who.int/news-room/fact-sheets/detail/suicide.

Zeeman, E. C. (1976). Catastrophe theory. Scientific American, 234, 65-83. https://doi.org/10.1038/scientificamerican0476-65 
Table 1. Suicidal Thoughts and Behaviour and Demographic Characteristics in Each Sample

\begin{tabular}{|c|c|c|c|}
\hline \multicolumn{2}{|c|}{ Characteristic } & $\begin{array}{l}\text { Sample 1 } \\
(\mathrm{N}=603)\end{array}$ & $\begin{array}{l}\text { Sample } 2 \\
(\mathrm{~N}=602)\end{array}$ \\
\hline \multicolumn{2}{|c|}{ Suicidal thought(s): lifetime presence } & $586(97.2 \%)$ & $589(97.8 \%)$ \\
\hline \multirow{4}{*}{ Recency } & Past month & $361(59.9 \%)$ & $353(58.6 \%)$ \\
\hline & Past year & $130(21.6 \%)$ & $132(21.9 \%)$ \\
\hline & $1-2$ years ago & $36(6 \%)$ & $47(7.8 \%)$ \\
\hline & $2+$ years ago & $59(9.8 \%)$ & $57(9.5 \%)$ \\
\hline \multicolumn{2}{|c|}{ Suicide attempt(s): lifetime presence } & $384(63.7 \%)$ & $385(64 \%)$ \\
\hline \multicolumn{2}{|c|}{$1-5$} & $261(43.3 \%)$ & $260(43.2 \%)$ \\
\hline \multirow{4}{*}{$\begin{array}{l}\text { Lifetime } \\
\text { frequency }\end{array}$} & $5-10$ & $66(10.9 \%)$ & $74(12.3 \%)$ \\
\hline & $10-15$ & $29(4.8 \%)$ & $2(3.7 \%)$ \\
\hline & $15+$ & $28(4.6 \%)$ & $29(4.8 \%)$ \\
\hline & Past month & $39(6.5 \%)$ & $58(9.6 \%)$ \\
\hline \multirow{3}{*}{ Recency } & Past year & $114(18.9 \%)$ & $113(18.8 \%)$ \\
\hline & $1-2$ years ago & $84(13.9 \%)$ & $53(8.8 \%)$ \\
\hline & $2+$ years ago & $147(24.4 \%)$ & $161(26.7 \%)$ \\
\hline \multirow[t]{3}{*}{ Age } & & $28.20(10.23)$ & $27.96(10.8)$ \\
\hline & Male & $45(7.5 \%)$ & $30(5 \%)$ \\
\hline & Female & $500(82.9 \%)$ & $503(83.6 \%)$ \\
\hline \multirow[t]{5}{*}{ Gender } & Transgender/Trans* & $20(3.3 \%)$ & $25(4.2 \%)$ \\
\hline & Non binary gender & $33(5.5 \%)$ & $40(6.6 \%)$ \\
\hline & Prefer not to say & $3(0.5 \%)$ & $3(0.5 \%)$ \\
\hline & White & $548(90.9 \%)$ & $549(91.2 \%)$ \\
\hline & $\begin{array}{l}\text { Mixed/Multiple ethnic } \\
\text { groups }\end{array}$ & $29(4.8 \%)$ & $24(4 \%)$ \\
\hline \multirow[t]{4}{*}{ Ethnicity } & $\begin{array}{l}\text { Asian (Indian, Pakistani, } \\
\text { Bangladeshi, Chinese) }\end{array}$ & $13(2.2 \%)$ & $12(2 \%)$ \\
\hline & Indian & $4(0.7 \%)$ & $3(0.5 \%)$ \\
\hline & Other ethnic group & $5(0.8 \%)$ & $10(2 \%)$ \\
\hline & Prefer not to say & $2(0.3 \%)$ & $4(0.7 \%)$ \\
\hline
\end{tabular}


Table 2. Taxometric Indicators and Properties in Each Sample

\begin{tabular}{|c|c|c|c|}
\hline Indicator & $\begin{array}{l}\text { Validity using } \\
\text { Cohen's } d\end{array}$ & Skew & Kurtosis \\
\hline \multicolumn{4}{|c|}{ Sample $1(N=603)$} \\
\hline $\begin{array}{l}\text { Current suicidal } \\
\text { ideation }\end{array}$ & 1.55 & 0.40 & -0.61 \\
\hline $\begin{array}{l}\text { Duration of } \\
\text { suicidal thoughts }\end{array}$ & 1.80 & -0.38 & -1.38 \\
\hline $\begin{array}{l}\text { Belief that suicide } \\
\text { is the only option }\end{array}$ & 1.26 & -0.19 & -1.41 \\
\hline \multicolumn{4}{|c|}{ Sample $2(N=602)$} \\
\hline $\begin{array}{l}\text { Current suicidal } \\
\text { ideation }\end{array}$ & 2.66 & 0.37 & -0.74 \\
\hline $\begin{array}{l}\text { Duration of } \\
\text { suicidal thoughts }\end{array}$ & 1.20 & -0.50 & -1.24 \\
\hline $\begin{array}{l}\text { Belief that suicide } \\
\text { is the only option }\end{array}$ & 1.33 & -0.12 & -1.49 \\
\hline
\end{tabular}



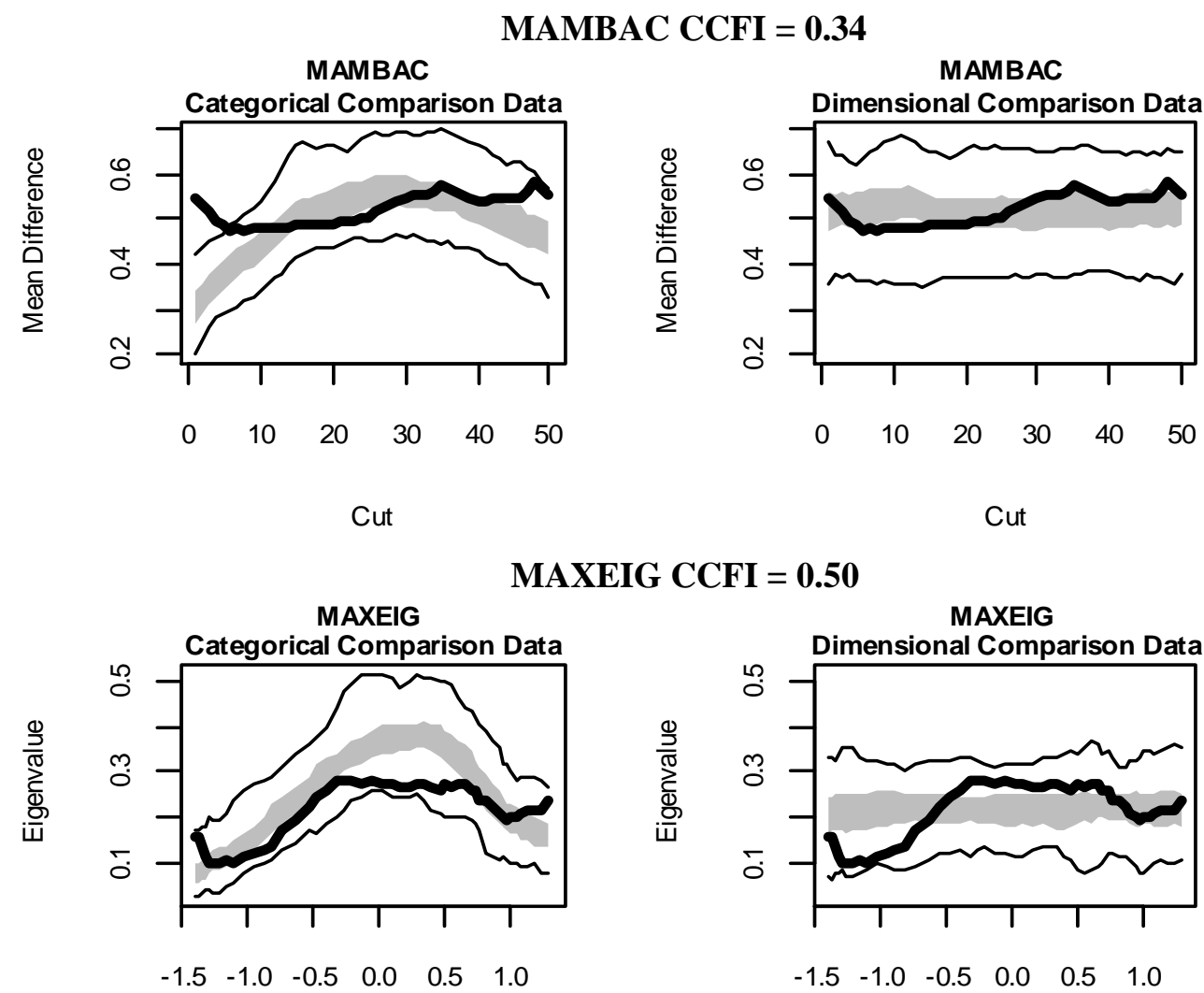

Cut

MAXEIG CCFI $=0.50$

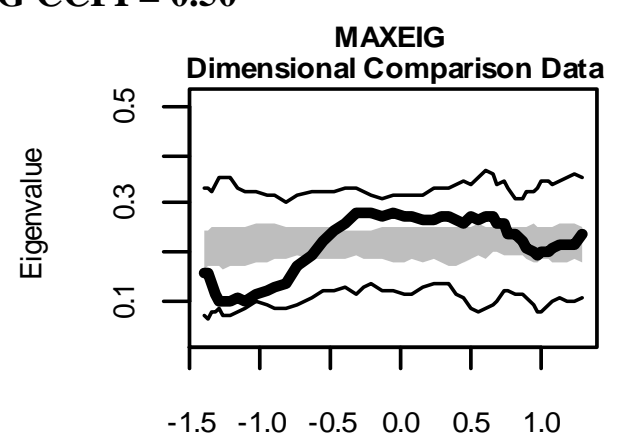

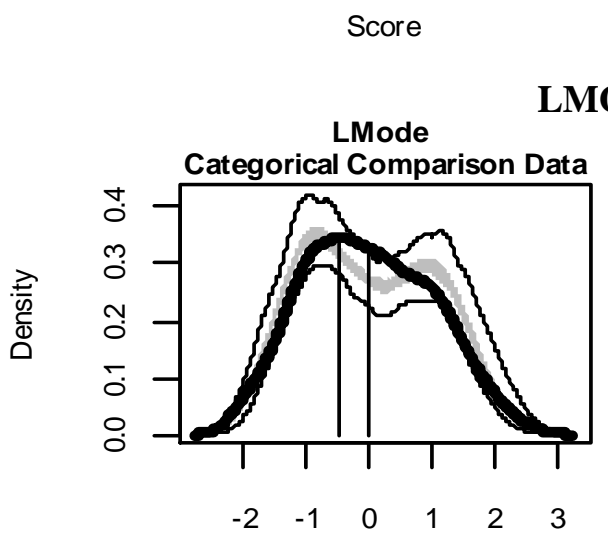

Factor Score
LMODE CCFI = $0.42 \quad$ LMode

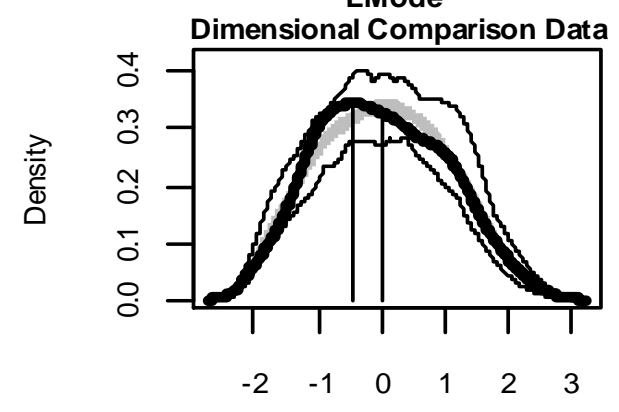

Factor Score

Figure 1. Results for each taxometric procedure in Sample 1. Dark lines show curves for empirical data and lighter lines show the minimum and maximum values for parallel analyses of 100 samples of comparison data. Shaded regions contain the middle $50 \%$ of values for parallel analyses of comparison data. CCFI = Comparison Curve Fit Index; MAMBAC = Mean Above Minus Below A Cut; MAXEIG = MAXimum EIGenvalue; L-Mode = Latent Mode. 
CCFI Profiles

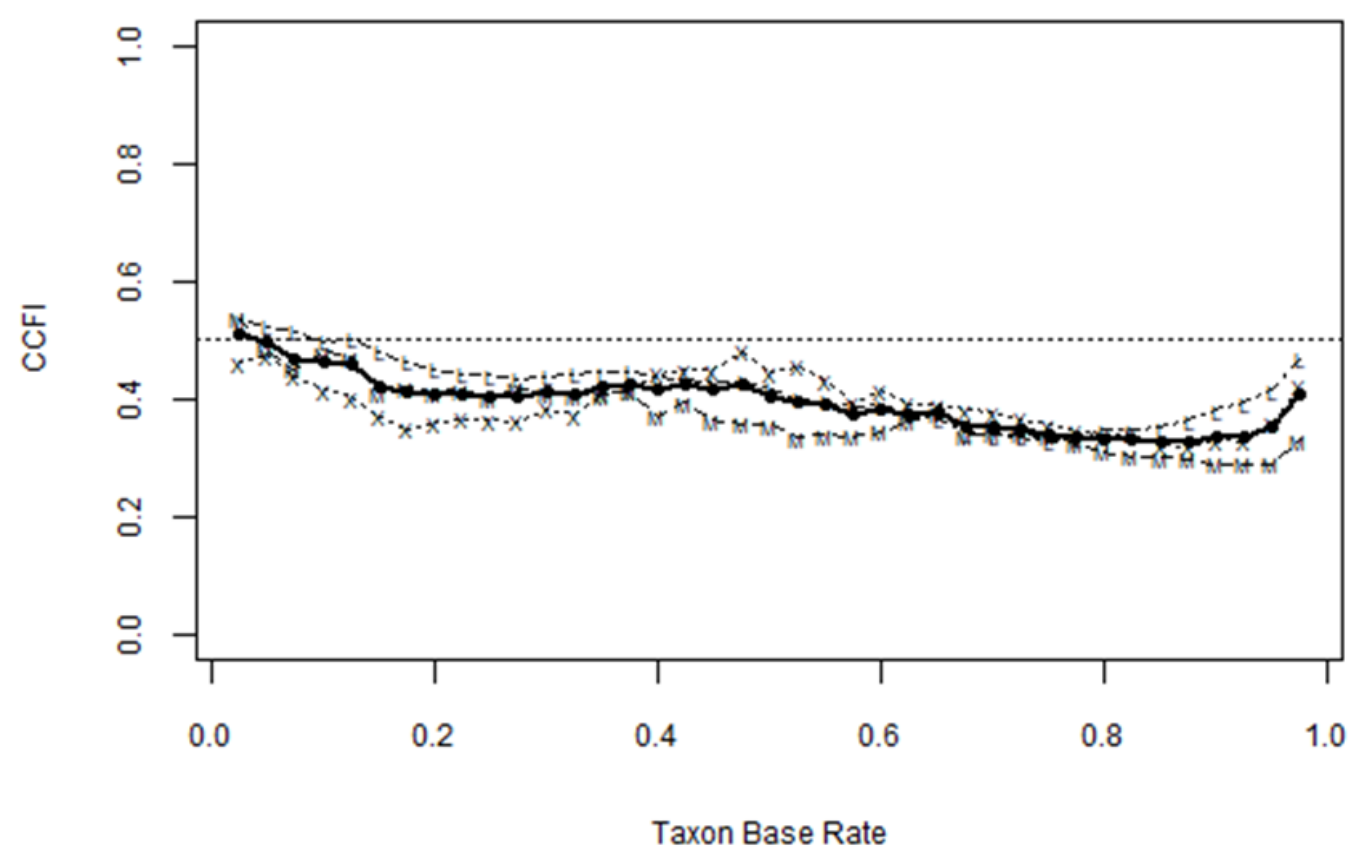

Figure 2. CCFI profile analysis for Sample 1. Three lines denote the results of the taxometric procedures used: M for MAMBAC (mean above minus below a cut), X for MAXEIG (MAXimum EIGenvalue), and L for L-Mode (latent mode). The darker, solid line indicates the average of the CCFIs from all three procedures. 

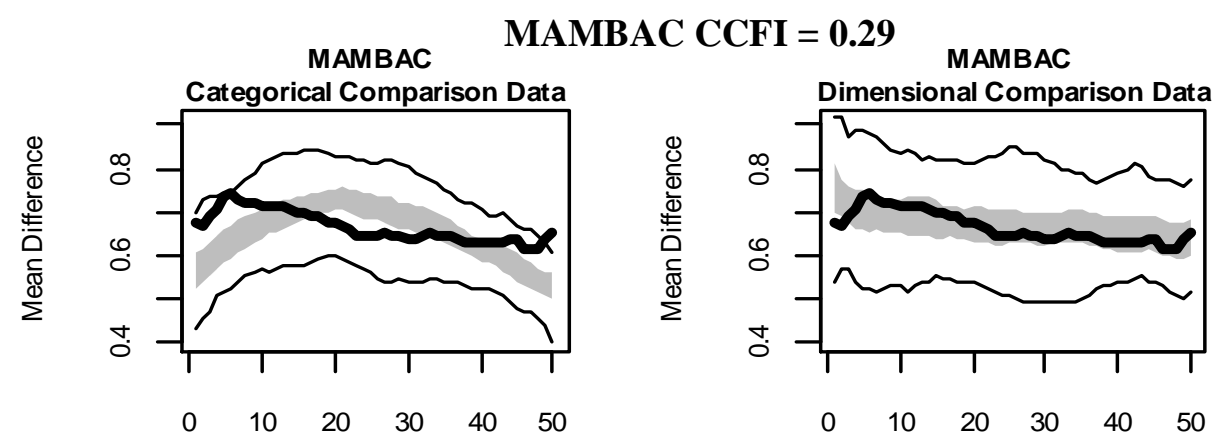

Cut

Cut
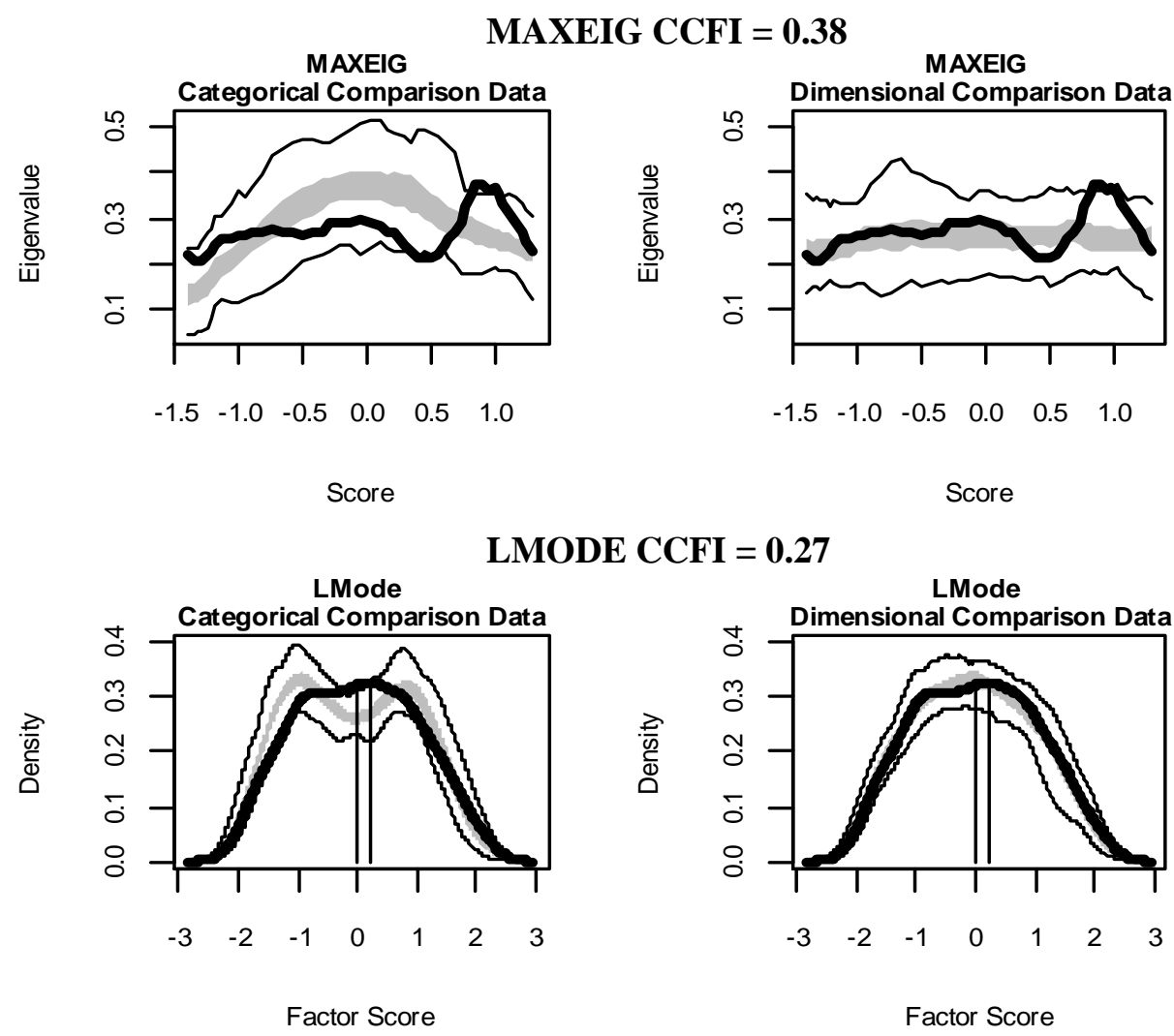

Score

MODE CCFI $=0.27$

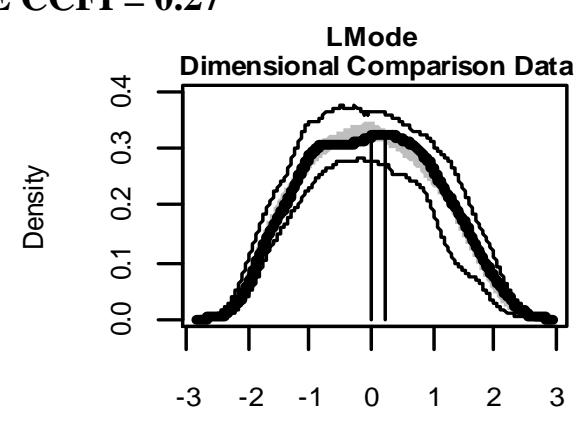

Factor Score

Figure 3. Results for each taxometric procedure in Sample 1. Dark lines show curves for empirical data and lighter lines show the minimum and maximum values for parallel analyses of 100 samples of comparison data. Shaded regions contain the middle $50 \%$ of values for parallel analyses of comparison data. CCFI = Comparison Curve Fit Index; MAMBAC = Mean Above Minus Below A Cut; MAXEIG = MAXimum EIGenvalue; L-Mode = Latent Mode. 


\section{CCFI Profiles}

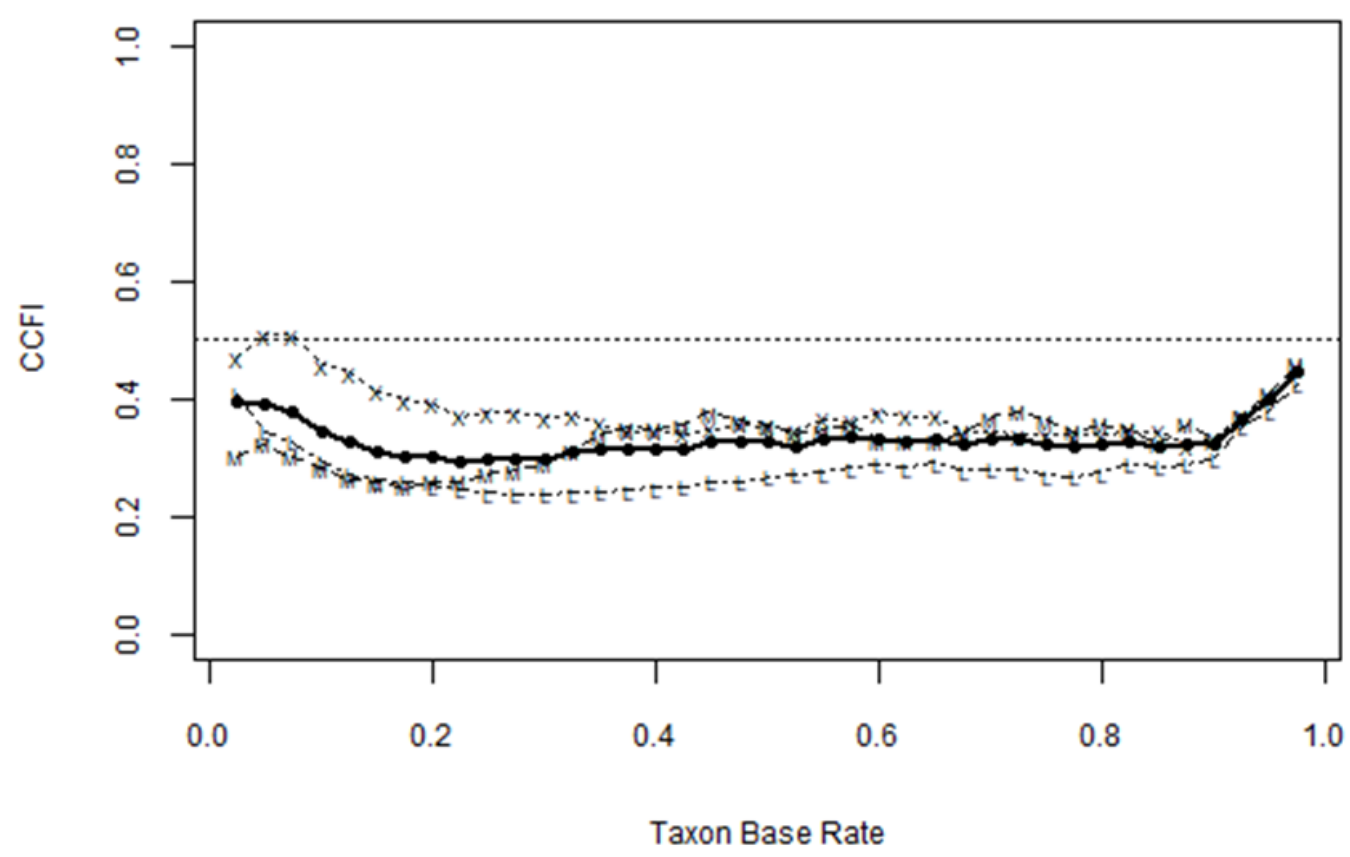

Figure 4. CCFI profile analysis for Sample 2. Three lines denote the results of the taxometric procedures used: M for MAMBAC (mean above minus below a cut), $\mathrm{X}$ for MAXEIG (MAXimum EIGenvalue), and L for L-Mode (latent mode). The darker, solid line indicates the average of the CCFIs from all three procedures. 